\title{
Overexpression of TIP30 inhibits the growth and invasion of glioma cells
}

\author{
YINGYING HU ${ }^{1,2}$, FENGSHENG CHEN ${ }^{1}$, FEIYE LIU ${ }^{1}$, XINHUI LIU $^{1}$, NA HUANG $^{1}$, \\ XIAOLI CAI ${ }^{1}$, YI SUN ${ }^{2}$, AIMIN LI $^{1}$ and RONGCHENG LUO ${ }^{1}$ \\ ${ }^{1}$ Cancer Center, Traditional Chinese Medicine-Integrated Hospital, Southern Medical University, Guangzhou, \\ Guangdong 510315; ${ }^{2}$ Cancer Center, 3rd People's Hospital, Dongguan, Guangdong 523326, P.R. China
}

Received March 16, 2014; Accepted June 4, 2015

DOI: $10.3892 / \mathrm{mmr} .2015 .4619$

\begin{abstract}
Glioma is an aggressive malignancy with limited effective treatment and poor prognosis. Therefore, the identification of novel prognostic markers and effective therapeutic targets is important for the treatment of human glioma. TIP30 is a tumor suppressor involved in the regulation of numerous cellular processes, including tumor cell growth, metastasis, and angiogenesis in various human cancers. The present study investigated whether Tat-interacting protein (TIP)30 was able to regulate tumorigenesis and predict the clinical outcome of patients with glioma. A total of 92 human glioma tissue samples and 10 normal brain tissue samples were examined by immunostaining. The results indicated that the expression levels of TIP30 significantly decreased in glioma tissue samples. as compared with normal brain tissue samples. Furthermore, TIP30 expression was inversely correlated with tumor histological classification, pathological grade, tumor size, and epidermal growth factor receptor (EGFR) expression; however, no association was detected between TIP30 expression and patient age and gender. In addition, patients with positive TIP30 expression exhibited significantly longer median overall survival rates, as compared with those with negative TIP30 expression. In vitro experiments revealed that upregulation of TIP30 expression by lentiviral vector transfection inhibited cell growth and induced cell apoptosis, as determined by MTT assay and Annexin V-fluorescein isothiocyanate staining, respectively. In addition, TIP30 expression markedly attenuated cell migration and invasion, as determined by wound healing and transwell assays. Upregulation of TIP30 expression in glioma cells decreased the expression levels of EGFR and its associated downstream molecules
\end{abstract}

Correspondence to: Dr Rongcheng Luo or Dr Aimin Li, Cancer Center, Traditional Chinese Medicine-Integrated Hospital, Southern Medical University, 13 Shiliugang Road, Guangzhou, Guangdong 510315, P.R. China

E-mail: luorc01@163.com

E-mail: liaimin2005@163.com

Key words: TIP30, epidermal growth factor receptor, glioma, prognosis, proliferation, metastasis phosphorylated extracellular signal-regulated kinases (ERK) and phosphorylated AKT, as determined by western blot analysis. The results of the present study indicated that TIP30 may suppress oncogenesis and glioma progression, thereby improving the prognosis of patients with glioma. Therefore, TIP30 may prove useful as a prognostic biomarker, and as a potential target for glioma therapy.

\section{Introduction}

Glioma is the most common type of malignant intracranial tumor, accounting for $\sim 50 \%$ of all intracranial tumors (1). Glioma is the second most common cause of cancer-associated mortality in patients $\leq 34$ years old, and the third most common cause in patients between 35 and 54 years old (2). Glioma is characterized by intense cell proliferation, diffuse infiltration, resistance to chemotherapy, high mortality, high recurrence, and poor clinical prognosis. Surgery and chemotherapy remain the standard treatments (3); however, prognosis is poor, and patients usually have a short survival time attributed to the aggressive nature of glioma. Therefore, identifying novel prognostic markers, carcinogenic pathways, and therapeutic targets is crucial for the treatment of glioma.

Tat-interacting protein (TIP) 30 is a $30 \mathrm{kD}$ molecule, which was initially identified as a tumor suppressor of small cell lung cancer (4). As a serine/threonine kinase and cofactor, TIP30 exhibits intrinsic kinase activity, and is able to phosphorylate the C-terminal domain of RNA polymerase II and potentiate human immunodeficiency virus transcription by binding to Tat $(5,6)$. TIP30 is widely expressed in normal human tissues, such as the heart, brain, lung, kidney, and pancreas, however its expression levels are decreased in numerous cancers such as breast cancer, lung cancer, liver cancer, gastric cancer, and colorectal cancer (7-11). Previous studies have demonstrated that TIP30 inhibits the development of numerous types of tumor by suppressing tumor cell growth, promoting cell apoptosis, inhibiting tumor angiogenesis, impairing DNA repair, regulating tumor cell glucose tolerance, and inhibiting nuclear transportation (12-16). However, the role of TIP30 in human glioma development remains to be elucidated.

Epidermal growth factor receptor (EGFR) is a transmembrane tyrosine kinase receptor of the ErbB family, which activates intracellular mitogenic signaling and other 
tumor-promoting cellular activities. EGFR is upregulated in $\sim 30 \%$ of gliomas (17). Humphrey et al (18) also reported that EGFR exhibited signs of amplification, rearrangement, mutation and overexpression in glioma tissue samples, which contributed to cancer progression. In addition, a recent study demonstrated that TIP30 functioned as a tumor suppressor by promoting the separation and endocytic degradation of the EGFR/EGF complex, and by terminating the signaling pathways of downstream components (19).

The present study aimed to investigate the expression and clinical significance of TIP30 in glioma tumors, as well as the correlation between TIP30 and EGFR.

\section{Materials and methods}

Tissue samples and histology. Paraffin-embedded cancerous tissue samples of 92 patients with glioma who underwent surgery between 2006 and 2011, were obtained from the Department of Cerebral Surgery of Nanfang Hospital (Guangzhou, China). The patients had not undergone chemotherapy or radiotherapy prior to surgery. Patient characteristics are shown in Table I. The glioma tissue samples were histologically classified by pathologists according to the World Health Organization criteria (20). A total of 10 normal brain tissue samples ( 3 females, 7 males; average age, 25.4 years old) were obtained from patients undergoing surgery for epilepsy (2000-2011) in order to serve as a control. All patients provided written informed consent, and all experiments were approved by the Research Ethics Committee of Nanfang Hospital (Guangzhou, China).

Immunohistochemistry (IHC). Slides containing the glioma tissue samples were deparaffinized and hydrated, prior to antigen retrieval and endogenous peroxidase clearance (Beyotime Institute of Biotechnology, Shanghai, China). Heat-induced antigen retrieval was performed in citrate buffer for $2 \mathrm{~min}$ at $100^{\circ} \mathrm{C}$. Endogenous peroxidase activity and nonspecific antigens were blocked with peroxidase blocking reagent containing $3 \%$ hydrogen peroxide and serum. The samples were then incubated with polyclonal antibodies targeting TIP30 (1:50; cat. no. ab22841; Abcam, Cambridge, UK) and EGFR (1:400; cat. no. 1902-1; Epitomics, Burlingame, CA, USA) at $4^{\circ} \mathrm{C}$ overnight. IHC images were captured using an Olympus fluorescence microscope (Olympus CKX41; Olympus Corporation, Tokyo, Japan) equipped with a camera.

The glioma tissue samples were reviewed by two pathologists. Staining was evaluated by measuring the intensity and percentage of positive cells in each sample. TIP30 staining was predominantly located in the cytoplasm, whereas EGFR staining was predominantly located in the cell membrane. Staining intensity was classified as 0 (negative), 1 (weak), 2 (moderate), or 3 (strong). An area of staining intensity was defined as 0 if $<10 \%, 1$ if $10-30 \%, 2$ if $31-60 \%$, and 3 if $>60 \%$. The staining percentage and intensity scores were multiplied in order to obtain a total score, of which $>2$ indicated a positive result.

Survival follow-up. The patients were followed-up by telephone until February, 9 2012. The median overall survival
Table I. Patient characteristics.

\begin{tabular}{lcc}
\hline Characteristic & No. of patients $(\mathrm{n}=92)$ & $\%$ \\
\hline Gender & & \\
$\quad$ Male & 40 & 43.5 \\
Female & 52 & 56.5 \\
Age (years) & & \\
$>35$ & 56 & 60.9 \\
$\leq 35$ & 36 & 39.1 \\
Histological classification & & \\
Astrocytomas & 42 & 45.7 \\
Oligodendrogliomas & 25 & 27.1 \\
Glioblastoma multiforme & 25 & 27.1 \\
Pathological grade & & \\
I-II & 42 & 45.7 \\
III-IV & 50 & 54.3 \\
Tumor size (cm) & & \\
$\geq 5$ & 47 & 48.9 \\
$<5$ & 45 & \\
\hline
\end{tabular}

(mOS) rates were calculated from the day of surgery until the follow-up deadline, or until the patients succumbed to their disease.

Cell culture and lentivirus infection. U87 glioma cells (American Type Tissue Collection, Manassas, VA, USA) were cultured in Dulbecco's modified Eagle's medium (DMEM) supplemented with 10\% fetal bovine serum (FBS) (Beyotime Institute of Biotechnology). Human TIP30 cDNA plasmids (obtained from Michigan State University, Lansing, MI, USA) were transfected into $293 \mathrm{~T}$ cells $\left(1 \times 10^{6}\right.$; obtained from Cell Biology Laboratory, Southern Medical University, Guangzhou, China) for $48 \mathrm{~h}$ to generate an lentivirus using Lipofectamine ${ }^{\circledR} 2000$ (Invitrogen Life Technologies, Carlsbad, CA, USA), according to the manufacturer's instructions. Control groups included the mock treatment group (Mock) and vector-transfected group ( $\mathrm{Lv}-\mathrm{Con})$. The viral supernatant was subsequently collected and used to infect the U87 glioma cells. Further analysis was performed $72 \mathrm{~h}$ post-transfection. Reverse transcription-quantitative polymerase chain reaction reverse transcription-quantitative polymerase chain reaction (RT-qPCR) and western blotting were performed in order to determine the efficiency of transfection.

$R T$-qPCR. RT-qPCR was used to quantify TIP30 mRNA expression. Total RNA from all cells were extracted using TRIzol reagent (Takara Bio, Inc., Otsu, Japan) and reverse transcribed to cDNA using the PrimeScript kit (Takara Bio, Inc.) according to the manufacturer's instructions. RT-qPCR was performed using Fast SYBR Green I kit (Takara Bio, Inc.) and an ABI 7500 thermal cycler (Applied Bioscience; Thermo Fisher Scientific, Inc., Waltham, MA, USA) was used. The following primers were used: TIP30 sense, 5'-AGAAACCGGCAGAGTGCTCTTA-3', antisense, 5'-GTACCCAGGCAACAGAATCCA-3'; and $\beta$-actin 
sense, 5'-TGGCACCCAGCACAATGAA-3', and antisense, 5'-CTAAGTCATAGTCCGCCTAGAAGCA-3'. PCR cycling conditions were as follows: Once cycle of $95^{\circ} \mathrm{C}$ for $30 \mathrm{sec}$, followed by 40 cycles of $95^{\circ} \mathrm{C}$ for $5 \mathrm{sec}, 60^{\circ} \mathrm{C}$ for $20 \mathrm{sec}$, and $65^{\circ} \mathrm{C}$ for $15 \mathrm{sec}$. Specificity of amplification products was confirmed by melting curve analysis. Each sample was assayed in triplicate in independent reactions. The expression of RNA was calculated using the $2^{-\Delta \Delta \mathrm{Ct}}$ method.

Western blotting. Briefly, the cells were washed twice with ice-cold phosphate-buffered saline and lysed in $250 \mathrm{ml}$ radioimmunoprecipitation assay buffer containing protease inhibitors (Beyotime Institute of Biotechnology). The protein samples were collected from the cell lysates, and the protein content of the supernatants was determined with a Bradford assay (Beyotime Institute of Biotechnology), using a protein assay dye reagent and bovine serum albumin (Invitrogen Life Technologies) as the standard. For each blot an internal standard was loaded alongside the protein samples $(40 \mu \mathrm{g})$, and the samples were separated using self-cast stacking $10 \%$ resolving gels in Tris-glycine running buffer. In transfer buffer, the blots were transferred wet onto polyvinylidene fluoride membranes (EMD Millipore, Billerica, MA, USA) for $90 \mathrm{~min}$ at $120 \mathrm{~V}$. Membranes were incubated with $1 \%$ fat-free milk for $1 \mathrm{~h}$ at room temperature with agitation, for blocking. Membranes were subsequently washed two times for $10 \mathrm{~min}$ in tris-buffered saline containing Tween (TBST) and then incubated with primary antibody overnight $(16 \mathrm{~h})$ at $4^{\circ} \mathrm{C}$. The primary antibodies were as follows: Anti-TIP30 (1:1,000; cat no. ab22841; Abcam), anti-EGFR (1:2,000, cat. no. 1902-1; Epitomics), anti-pAKT (1:1,000, cat. no. 5056, Cell Signaling Technology Inc.), anti-pERK (1:1,000, cat. no. 4370P, Cell Signaling Technology Inc.). The following morning, the membranes were washed a further two times (10 min) in TBST, and incubated with a secondary antibody (HRP-labeled goat anti-rabbit IgG; 1:1000; cat. no. A0208; Beyotime Institute of Biotechnology) in $1 \%$ fat-free milk for $60 \mathrm{~min}$ at room temperature. Following a final two washes $(10 \mathrm{~min})$ in TBST, the membranes were visualized using Odyssey Infrared Laser-Imaging system (LI-COR Biosciences, Lincoln, NE, USA).

Cell proliferation assay. An MTT assay was used to evaluate cell proliferation. Cells were seeded in 96-well plates $\left(1 \times 10^{3}\right.$ cells/well) and observed for 7 days. A total of $10 \mu 1 \mathrm{MTT}$ (Beyotime Institute of Biotechnology) was added to each well and incubated at $37^{\circ} \mathrm{C}$ for $4 \mathrm{~h}$. Subsequently, $150 \mu \mathrm{l}$ dimethyl sulfoxide (Beyotime Institute of Biotechnology) was added to the cells and the cells were agitated for $15 \mathrm{~min}$. The optical density values of each well were measured at a wavelength of $490 \mathrm{~nm}$ (Thermo Fisher Scientific, Inc.). All experiments were performed in triplicate.

Cell apoptosis assay. The U87 cells $\left(1 \times 10^{5}\right)$ were harvested through trypsinization (Invitrogen Life Technologies) and resuspended in Annexin V-fluorescein isothiocyanate (FITC) binding buffer (Beyotime Institute of Biotechnology). The Annexin V-FITC solution was then incubated at room temperature in the dark for $10 \mathrm{~min}$. The solution was centrifuged at $1,000 \mathrm{x} \mathrm{g}$ for $5 \mathrm{~min}$ at $4^{\circ} \mathrm{C}$ and the supernatant was discarded. Annexin V-FITC binding buffer and propidium iodide (Beyotime Institute of Biotechnology) were added to the U87 cells, and fluorescence-activated cell sorting analysis was performed following $1 \mathrm{~h}$ using a FACSCalibur (BD Biosciences, San Jose, CA, USA).

Cell migration and invasion assay. The U87 cells $\left(1 \times 10^{5}\right)$ were seeded in a 6-well plate and a wound was made using a plastic pipette tip. Images were captured at 0,18 and $36 \mathrm{~h}$. Transwell chambers containing polycarbonate membrane filters ( $8 \mu \mathrm{m}$ pore size; BD Biosciences) were coated with Matrigel. A total of $0.2 \mathrm{ml}$ DMEM was added to the upper chamber containing the glioma cells, and $0.6 \mathrm{ml}$ complete medium supplemented with $15 \%$ FBS was added to the lower chamber. Following a $48 \mathrm{~h}$ time period, the cells that had migrated through the membranes were fixed in paraformaldehyde and stained with $0.1 \%$ crystal violet (Beyotime Institute of Biotechnology). The images of five randomly selected fields were captured using a microscope (Olympus CKX41, Olympus Corporation) in order to calculate the number of cells that had successfully invaded the Matrigel (BD Biosciences).

Statistical analysis. SPSS 13.0 software (SPSS, Inc., Chicago, IL, USA) was used to carry out all statistical analyses. Pearson $\chi^{2}$ tests were used to evaluate the expression levels of TIP30 in the glioma tissue samples, as compared with normal brain tissue samples, and to determine the association between TIP30 expression and clinicopathological features. The overall survival time was analyzed using the Kaplan-Meier method, and a Cox regression model was used for multvariate analysis of the factors influencing mOS. All other in vitro assay results were presented as the mean \pm standard deviation, and a Student's t-test was used to determine statistically significant differences between groups. $\mathrm{P}<0.05$ was considered to indicate a statistically significant difference. All statistical tests were two-way analyses.

\section{Results}

Expression levels of TIP30 and EGFR and their correlation with the clinicopathological features of glioma. IHC was performed in order to assess the expression of TIP30 in glioma tissue samples, as compared with normal brain tissue samples. TIP30-positive immunostaining was present in $90 \%(9 / 10)$ of normal brain tissue samples, which was significantly higher than the $54.3 \%$ (50/92) present in glioma tissue samples $(\mathrm{P}=0.03)$. Conversely, positive expression of EGFR in glioma tissue samples was significantly higher, as compared with normal brain tissue samples (57.6 and 20.0\%, respectively; $\mathrm{P}=0.04$ ) (Table II) (Figs. $1 \mathrm{~A}$ and $\mathrm{B}$ ).

There was no significant correlation between TIP30 expression, and age and gender. However, TIP30 expression was inversely correlated with histological classification, pathological grade, and tumor size. Conversely, EGFR expression was positively correlated with histological classification, pathological grade, and tumor size (Table II). Following recent research on TIP30-induced regulation of EGFR degradation in normal and cancerous cells (19), the correlation between TIP30 and EGFR expression was further investigated using 
Table II. Correlation between the protein expression of Tat-interacting protein (TIP)30 and epidermal growth factor receptor (EGFR), and the clinicopathological features of patients with glioma.

\begin{tabular}{|c|c|c|c|c|c|c|c|}
\hline \multirow[b]{2}{*}{ Variable } & \multirow[b]{2}{*}{$\mathrm{n}$} & \multicolumn{3}{|c|}{ TIP30 } & \multicolumn{3}{|c|}{ EGFR } \\
\hline & & Positive & Negative & P-value & Positive & Negative & P-value \\
\hline Tissue & & & & 0.03 & & & 0.04 \\
\hline Glioma & 92 & 50 & 42 & & 53 & 39 & \\
\hline Normal brain & 10 & 9 & 1 & & 2 & 8 & \\
\hline Gender & & & & 0.76 & & & 0.21 \\
\hline Male & 40 & 21 & 19 & & 26 & 14 & \\
\hline Female & 52 & 29 & 23 & & 27 & 25 & \\
\hline Age & & & & 0.14 & & & 0.33 \\
\hline$>35$ years & 56 & 27 & 29 & & 30 & 26 & \\
\hline$\leq 35$ years & 36 & 23 & 13 & & 23 & 13 & \\
\hline Histology & & & & 0.03 & & & 0.03 \\
\hline Astrocytomas & 42 & 27 & 15 & & 20 & 22 & \\
\hline Oligodendrogliomas & 25 & 15 & 10 & & 13 & 12 & \\
\hline Glioblastoma multiforme & 25 & 8 & 17 & & 20 & 5 & \\
\hline Pathological grade & & & & 0.03 & & & 0.01 \\
\hline I-II & 42 & 28 & 14 & & 18 & 24 & \\
\hline III-IV & 50 & 22 & 28 & & 35 & 15 & \\
\hline Tumor size $(\mathrm{cm})$ & & & & 0.02 & & & 0.01 \\
\hline$\geq 5$ & 47 & 20 & 27 & & 33 & 14 & \\
\hline$<5$ & 45 & 30 & 15 & & 20 & 25 & \\
\hline
\end{tabular}

the Spearman's Rank Correlation Coefficient analysis. Notably, the protein expression of TIP30 was inversely correlated with the protein expression of EGFR in glioma tissue samples (Table III).

The mOS time of all patients was 23.7 months. Using the Kaplan-Meier method, previous determined factors including glioblastoma multiforme histology and pathological grades III-IV were shown to be associated with a shorter mOS time. Notably, patients with TIP30-positive expression or EGFR-negative expression exhibited significantly longer mOS times (26.4 and 27.0 months, respectively), as compared with those with TIP30-negative or EGFR-positive expression (26.4 and 27.0, vs. 20.6 and 21.3 months, respectively; $\mathrm{P}<0.05$; Fig. 1C and Table IV). To determine whether TIP30 expression was an independent predictor of patient mOS, a Cox regression model was used for the multivariate analysis of all of the factors influencing mOS. The results indicate that TIP30 expression, alongside histological classification and pathological grade, were independent prognostic factors for cancer-specific mOS (Table V).

Overexpression of TIP30 inhibits glioma cell growth. In order to elucidate the functional role of TIP30 in glioma cells, TIP30 was overexpressed in U87 glioma cells, which previously possessed low expression levels of TIP30 (8), using a lentivirus encoding TIP30 cDNA (LV-TIP30). As determined by RT-qPCR and western blotting, the cells in the LV-TIP30 group stably overexpressed TIP30 at both the mRNA and protein level, as compared with the mock treatment group
Table III. Correlation between the protein expression of Tat-interacting protein (TIP)30 and epidermal growth factor receptor (EGFR) in glioma tissue samples.

\begin{tabular}{lccccc}
\hline & \multicolumn{2}{c}{ TIP30 (no. of cases) } & & \\
\cline { 2 - 3 } EGFR & Positive & Negative & & r-value & P-value \\
\hline Positive & 16 & 37 & & \\
Negative & 34 & 5 & & $<0.01$ \\
\hline
\end{tabular}

(Mock) or vector-transfected group (LV-Con) (Fig. 2A and 2B). As demonstrated by the cell proliferation assay, upregulation of TIP30 markedly inhibited the proliferation of TIP30-transfected cells, as compared with the cells from the Mock and LV-Con groups (Fig. 2C). Annexin V-FITC staining demonstrated that the percentage of early apoptotic cells was significantly increased in the LV-TIP30 U87 cells, as compared with the LV-Con and mock U87 cells $(36.2 \pm 3.88,0.4 \pm 0.13$ and $0.84 \pm 0.16 \%$, respectively; $\mathrm{P}<0.05)$. These results indicate that TIP30-induced U87 glioma cell growth inhibition is likely due to the activation of early apoptosis (Fig. 2D and 2E).

Overexpression of TIP30 inhibits invasion and migration of glioma cells. As determined by the wound healing assay, the 
Table IV. Univariate analysis of individual parameters correlated with the median overall survival (mOS) of patients with glioma.

\begin{tabular}{|c|c|c|c|}
\hline Variable & $\mathrm{n}$ & mOS (months) & P-value \\
\hline \multicolumn{4}{|l|}{ Gender } \\
\hline Male/female & $40 / 52$ & $25.5 / 22.4$ & 0.10 \\
\hline \multicolumn{4}{|l|}{ Age } \\
\hline$>35 / \leq 35$ years & $56 / 36$ & $24.321 / 22.833$ & 0.32 \\
\hline \multicolumn{4}{|l|}{ Histological classification } \\
\hline Astrocytomas/oligodendrogliomas/GBM & $42 / 25 / 25$ & $27.8 / 25.2 / 15.480$ & $<0.01$ \\
\hline \multicolumn{4}{|l|}{ Pathological grade } \\
\hline I-II/III-IV & $42 / 50$ & $28.5 / 19.7$ & $<0.01$ \\
\hline \multicolumn{4}{|l|}{ Tumor size } \\
\hline$\geq 5 \mathrm{~cm} /<5 \mathrm{~cm}$ & $47 / 45$ & $23.5 / 24.0$ & 0.48 \\
\hline \multicolumn{4}{|l|}{ TIP30 expression } \\
\hline Positive/negative & $50 / 42$ & $26.4 / 20.6$ & 0.02 \\
\hline \multicolumn{4}{|l|}{ EGFR expression } \\
\hline Positive/negative & $53 / 39$ & $21.3 / 27.0$ & 0.02 \\
\hline
\end{tabular}

TIP30, Tat-interacting protein 30, EGFR, epidermal growth factor receptor; GBM, glioblastoma multiforme.

Table V. Multivariate analysis of individual parameters correlated with overall rate (Cox-proportional hazards model).

\begin{tabular}{lcc}
\hline Variable & Hazard ratio & Confidence interval (95\%) \\
\hline $\begin{array}{l}\text { Histological classification } \\
\text { Astrocytomas/oligodendrogliomas/GBM }\end{array}$ & 1.474 & $1.081-2.011$ \\
$\begin{array}{l}\text { Pathological grade } \\
\text { I-II/III-IV }\end{array}$ & 2.825 & $1.566-5.098$ \\
$\begin{array}{l}\text { Tat-interacting protein (TIP)30 expression } \\
\text { Positive/negative }\end{array}$ & 1.823 & 0.014 \\
\hline
\end{tabular}

GBM, glioblastoma multiforme.

U87 glioma cells infected with LV-TIP30 migrated and filled the wound less rapidly, as compared with the LV-Con U87 glioma cells (Fig. 3A). Concordant with the wound healing assay results, the number of LV-TIP30 U87 glioma cells that invaded the Matrigel was significantly lower, as compared with the LV-Con group (62.2 \pm 8.3 , vs. $190.4 \pm 16.0$ cells per field; Fig. 3B and C). These data indicate that TIP30 may inhibit the invasion and migration of U87 glioma cells.

U87 glioma cell infection with LV-TIP30 induces downregulation of EGFR/phosphorylated (p)ERK/pAKT expression. EGFR expression is negatively correlated with TIP30 expression in glioma tissue samples, as previously mentioned. In addition, the overexpression of TIP30 in U87 glioma cells significantly downregulated the expression levels of EGFR in vitro, as determined by western blotting. Furthermore, the expression levels of pERK and pAKT were also markedly decreased following TIP30 overexpression (Fig. 4). These results suggest that upregulation of TIP30 may negatively regulate the expression of EGFR and its downstream signal pathways, resulting in the inhibition of tumor growth and invasion.

\section{Discussion}

To our knowledge, the present study is the first to report that TIP30 expression is markedly decreased in glioma tissue samples. The results demonstrated that TIP30 expression is inversely correlated with tumor histological classification, pathological grade, tumor burden, and EGFR expression. Reduced TIP30 expression also significantly affected the clinical outcome of patients with glioma, and predicted poor survival rate. Furthermore, the results indicated that TIP30 was an independent prognostic factor for the survival time of patients with glioma. In vitro experiments demonstrated that the overexpression of TIP30 is able to inhibit glioma cell growth, induce tumor cell apoptosis, and suppress tumor cell migration and invasion. In addition, overexpression of TIP30 
A

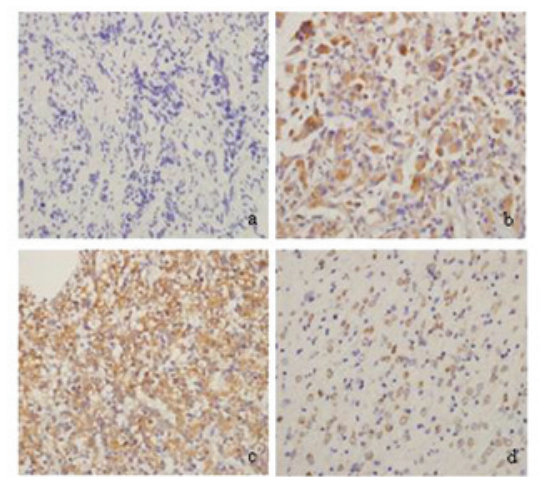

B

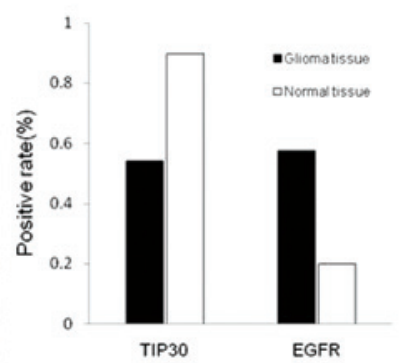

C
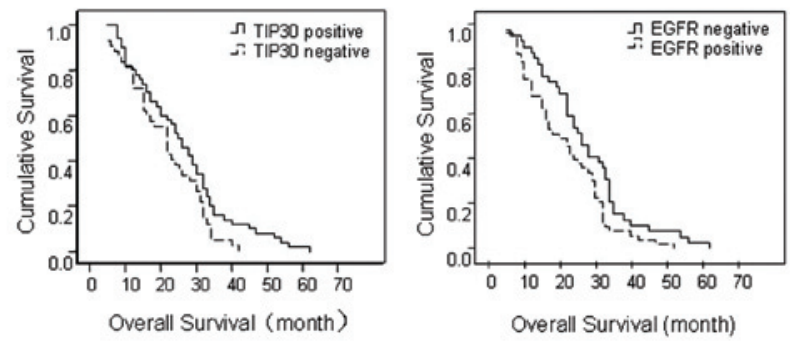

Figure 1. Expressions of Tat-interacting protein (TIP) 30 and epidermal growth factor receptor (EGFR) in glioma and normal brain tissue samples. (A) (a) Negative expression of TIP30 in glioma tissue samples. (b) Positive expression of TIP30 in normal tissue samples. (c) Strong positive expression of EGFR in glioma tissue samples. (d) Weak positive expression of epidermal growth factor receptor (EGFR) in normal brain tissue samples. (Magnification, $\mathrm{x} 400$ ) (B) Protein expression of TIP30 and EGFR in glioma and normal brain tissue samples (P<0.05). (C) Median overall survival (mOS) of patients with TIP30 and EGFR-positive and TIP30 and EGFR-negative expression. (Left panel) Patients with TIP30-positive expression exhibited longer mOS times, as compared with patients with TIP30-negative expression ( $\mathrm{P}=0.019)$, and (right panel) patients with EGFR-positive expression exhibited shorter mOS times, as compared with patients with EGFR-negative expression $(\mathrm{P}=0.021)$.

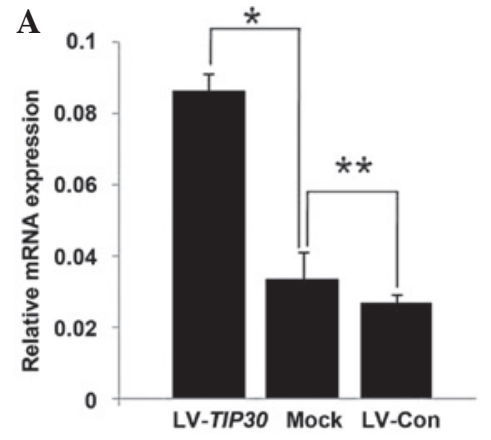

B

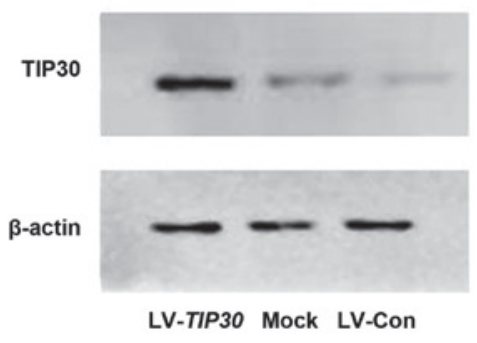

U87

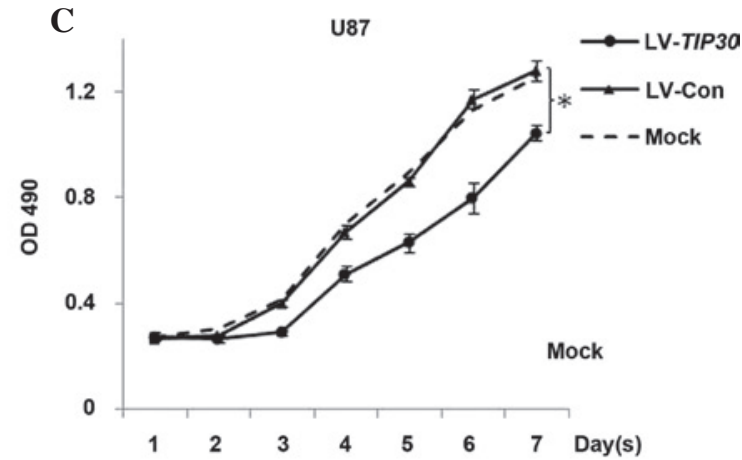

D

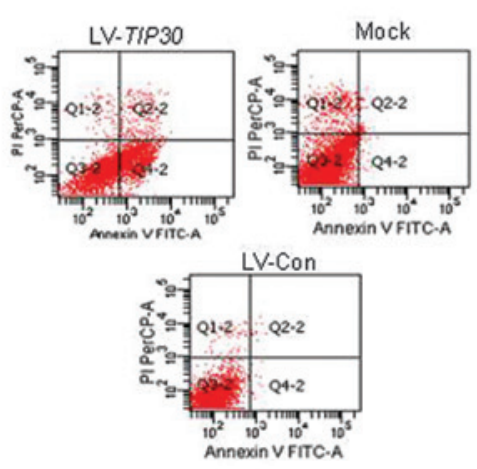

$\mathbf{E}$

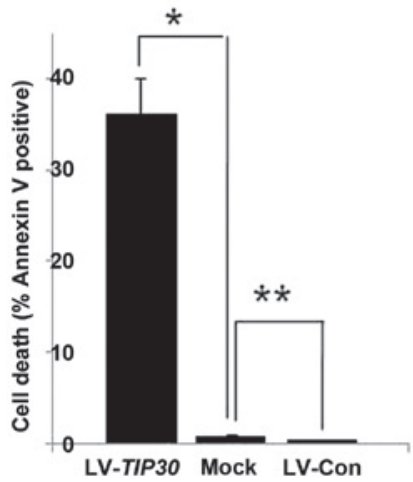

Figure 2. Overexpression of Tat-interacting protein (TIP)30 inhibits glioma cell growth. (A) mRNA expression levels of TIP30 were analyzed by real time-quantitative polymerase chain reaction in lentivirus (LV)-TIP30, mock and LV-control (Con) U87 glioma cells. (B) Protein expression levels of TIP30 were analyzed by western blotting. (C) Overexpression of TIP30 inhibits glioma cell growth. Four days following virus infection, the U87 cells were seeded in 96-well plates in order to carry out an MTT assay. "P<0.05 LV-TIP30 group, vs. the LV-Con group. (D) Cell apoptosis was detected by flow cytometry. The cells were assessed by flow cytometry following Annexin V staining. Q4-2, early apoptotic cells positively stained for Annexin V-fluorescein isothiocyanate (FITC), and negative for propidium iodide (PI); Q3-2, normal cells not stained by Annexin V-FITC or PI; Q2-2, necrotic cells and late apoptotic cells stained by both Annexin V-FITC and PI; (E) Data are presented as the mean \pm standard deviation of three independent experiments. ${ }^{*} \mathrm{P}<0.05$ and ${ }^{* *} \mathrm{P}>0.05$. 
A

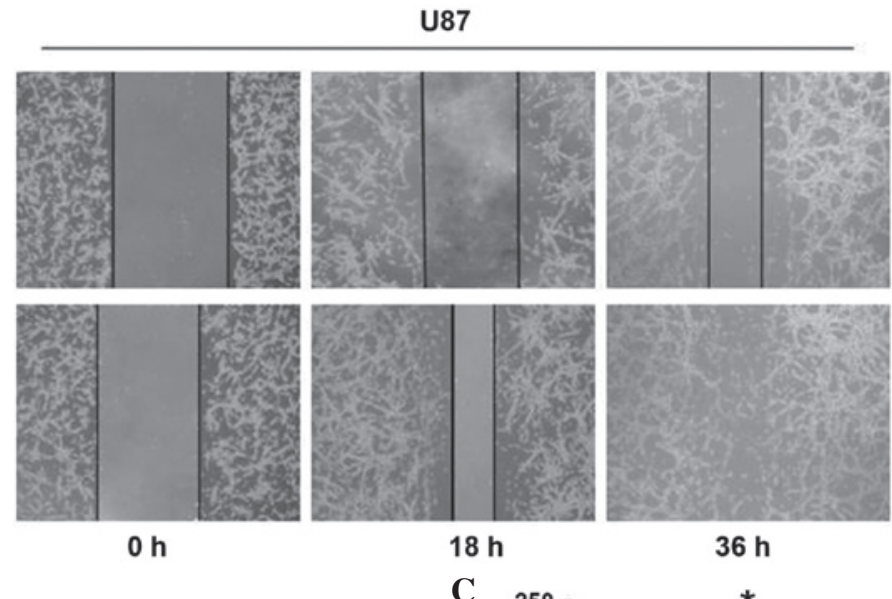

B

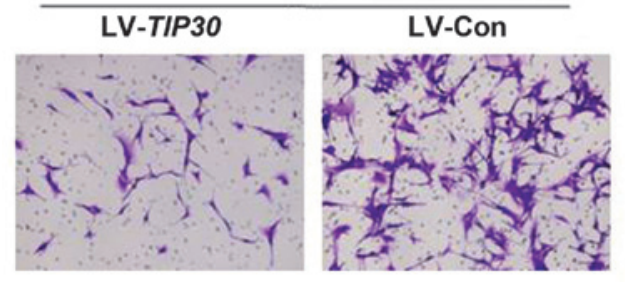

C

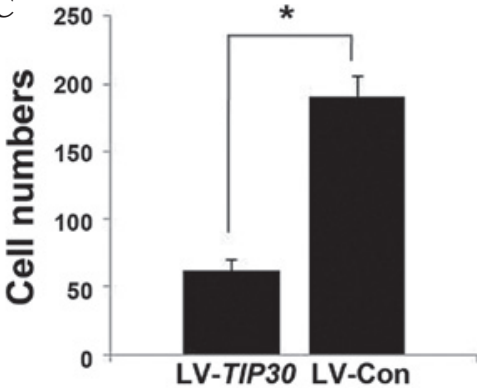

Figure 3. Overexpression of Tat-interacting protein (TIP)30 inhibits the migration and metastasis of U87 glioma cells. (A) U87 cells infected with lentivirus (LV)-TIP30 and LV-control (Con) were examined in a wound healing assay. Images were captured at 0, 18, and 36 h. (B) U87 cells infected with LV-TIP30 and LV-Con were incubated in Matrigel for $48 \mathrm{~h}$, and the number of cells invading the membrane was calculated from five randomly selected microscopic fields. (C) The number of cells invading through the Matrigel are presented as the mean \pm standard deviation. ${ }^{*} \mathrm{P}<0.05$.

in U87 glioma cells was able to induce the downregulation of EGFR expression and its downstream signaling components. These results indicated that TIP30 may regulate anti-carcinogenic activities in glioma cells.

The majority of gliomas are incurable and patient prognosis is usually poor. Histological classification and pathological grades are widely used as prognostic factors for patients with glioma. However, patients with similar histological or pathological grades may have different clinical outcomes. It is therefore necessary to identify novel prognostic and predictive markers. The results of the present study demonstrated that loss of TIP30 expression in patients with glioma was correlated with poor mOS. TIP30 expression, as well as histological classification and pathological grading, were shown to be successful independent prognostic factors for predicting the clinical outcome of patients with glioma. TIP30 expression was also markedly decreased in glioma tissue samples, as compared with normal brain tissue samples. These data indicated that loss of TIP30 expression may be involved in the development and progression of gliomas; however the intrinsic mechanism underlying the association between TIP30 and glioma has yet to be elucidated.

In vitro studies demonstrated that overexpression of TIP30 is able to inhibit glioma cell growth, which may be associated with TIP30-induced cell apoptosis. TIP30 was also shown to inhibit tumor cell migration and invasion in wounds and Matrigel in vitro. TIP30 is a putative tumor suppressor gene, which can downregulate genes associated with cell proliferation, apoptosis, and metastasis, thereby exhibiting anti-tumor effects in cancer (6). TIP30 is able to induce cellular apoptosis by elevating

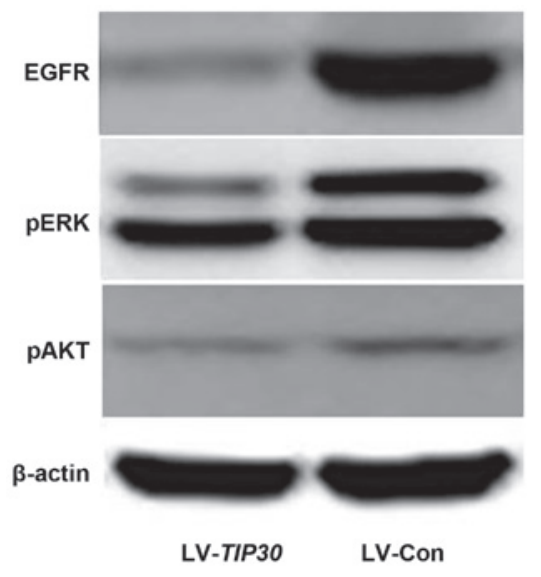

Figure 4. U87 glioma cell infection with lentivirus (LV)-Tat-interacting protein (TIP)30 induced significant downregulation of epidermal growth factor receptor (EGFR)/phosphorylated (p) extracellular signal-regulated kinases (ERK)/pAKT. The expression levels of EGFR, pERK, and pAKT were examined by western blotting in LV-TIP30 and LV-control (Con) U87 cells.

the expression levels of B cell lymphoma 2 (Bcl-2)-associated agonist of cell death, Siva, NM23-H2, Bcl-2-associated protein $\mathrm{X}$, and $\mathrm{p} 53$, promoting mitochondrial cytochrome $c$ release, and by downregulating the expression levels of anti-apoptotic genes such as Bcl-xL and c-myc (5,21). In addition, TIP30 inhibits the expression levels of tumor metastasis-associated genes, such as osteopontin (22) and calcium adhesion proteins in liver cancer (23). These results indicated that TIP30 may exhibit anti-tumor effects in glioma; however, the mechanism underlying TIP30 function in glioma remains to be elucidated. 
EGFR is commonly overexpressed and amplified in gliomas, which contributes to the uncontrolled proliferation and invasion of glioma cells (24). The results of the present study indicated that patients with glioma with positive EGFR expression exhibited significantly shorter mOS times, as compared with those with negative EGFR expression. Notably, the clinical data of the present study also demonstrated the expression levels of TIP30 were inversely correlated with the expression levels of EGFR in glioma tissue samples. Further in vitro studies demonstrated that the overexpression of TIP30 in glioma cells decreased the expression levels of EGFR, and its downstream signaling molecules. The correlation between TIP30 and EGFR has been the subject of numerous studies. Zhang et al (25) demonstrated that TIP30 knockout mice exhibited a higher incidence of spontaneous mammary tumors, which was correlated with the upregulation of EGFR and its intracellular signaling pathways pAKT and pERK. Zhang et al (26) also identified a protein complex consisting of TIP30, acyl-coenzyme A synthetase long-chain family member 4, and endophilin B1 that was able to promote the fusion of endocytic vesicles with Rab5a vesicles, thus promoting the exit of EGFR-EGF, acceleration of EGFR degradation, and termination of downstream signal transduction pathways such as phosphoinositide 3-kinase/ERK. The results of previous studies combined with those of the present study led to the hypothesis that TIP30 may exert its role, at least partly, through the regulation of EGFR and its downstream signaling pathways, resulting in the inhibition of tumor cell growth and metastasis in gliomas. However, the molecular mechanism underlying the interaction between TIP30 and EGFR in gliomas requires further research.

In conclusion, the results of the present study demonstrated that the expression of TIP30 is markedly decreased in glioma tissue samples. TIP30 may function as a tumor suppressor gene and act as a predictive factor for the prognosis of patients with glioma. Given that the overexpression of TIP30 in glioma cells inhibits tumor proliferation, metastasis and migration, TIP30 may be a potent therapeutic target for patients with glioma.

\section{Acknowledgements}

The authors of the present study are grateful to the Department of Pathology of Nanfang Hospital for providing the pathological tissue samples, and for the technical assistance and support in pathologic examinations; and to the Department of Physiology, Michigan State University for providing TIP30 cDNA plasmids. This study was supported by grants from the National Natural Science Foundation of China (no. 81372153) and the Natural Science Foundation of Guangdong Province (no. 2015A030313261).

\section{References}

1. Nygaard SJ, Haugland HK, Laerum OD, Lund-Johansen M, Bjerkvig R and Tysnes OB: Dynamic determination of human glioma invasion in vitro. J Neurosurg 89: 441-447, 1998.

2. Wen PY and Kesari S: Malignant gliomas in adults. N Engl J Med 395: 492-507, 2008.

3. Stupp R, Hegi ME, van den Bent MJ, Mason WP, Weller M, Mirimanoff RO, Cairncross JG, European Organisation for Research and Treatment of Cancer Brain Tumor and Radiotherapy Groups and National Cancer Institute of Canada Clinical Trials Group: Changing paradigms - an update on the multidisciplinary management of malignant glioma. Oncologist 11: 165-180, 2006
4. Shtivelman E: A link between metastasis and resistance to apoptosis of variant small cell lung carcinoma. Oncogene 14: 2167-2173, 1997.

5. Xiao H, Palhan V, Yang Y and Roeder RG: TIP30 has an intrinsic kinase activity required for up-regulation of a subset of apoptotic genes. EMBO J 19: 956-963, 2000.

6. Xiao H, Tao Y, Greenblatt J and Roeder RG: A cofactor, TIP30, specifically enhances HIV-1 Tat-activated transcription. Proc Natl Acad Sci USA 95: 2146-2151, 1998.

7. Huang QD, Hu XQ, Wan L, Gao GH, Zheng SR, You J and Guo GL: Clinical significance of CC3/TIP30 expression in breast carcinoma and its correlation with HER-2/neu. Zhonghua Wai Ke Za Zhi 50: 57-61, 2012 (In Chinese).

8. Tong X, Li K, Luo Z, Lu B, Liu X, Wang T, Pang M, Liang B, Tan M, Wu M, et al: Decreased TIP30 expression promotes tumor metastasis in lung cancer. Am J Pathol 174: 1931-1939, 2009.

9. Lu B, Ma Y, Wu G, Tong X, Guo H, Liang A, Cong W, Liu C, Wang H, Wu M, et al: Methylation of Tip30 promoter is associated with poor prognosis in human hepatocellular carcinoma. Clin Cancer Res 14: 7405-7412, 2008.

10. Li X, Zhang Y, Cao S, Chen X, Lu Y, Jin H, Sun S, Chen B, Liu J, Ding J, et al: Reduction of TIP30 correlates with poor prognosis of gastric cancer patients and its restoration drastically inhibits tumor growth and metastasis. Int J Cancer 124: 713-721, 2009.

11. Chen X, Cao X, Dong W, Luo S, Suo Z and Jin Y: Expression of TIP30 tumor suppressor gene is down-regulated in human colorectal carcinoma. Dig Dis Sci 55: 2219-2226, 2010.

12. Shi M, Zhang X, Wang P, Zhang HW, Zhang BH and Wu MC: TIP30 regulates apoptosis-related genes in its apoptotic signal transduction pathway. World J Gastroenterol 11: 221-227, 2005.

13. NicAmhlaoibh R and Shtivelman E: Metastasis suppressor CC3 inhibits angiogenic properties of tumor cells in vitro. Oncogene 20: 270-275, 2001.

14. Fong S, King F and Shtivelman E: CC3/TIP30 affects DNA damage repair. BMC Cell Biol 11: 23, 2010.

15. Sonveaux P: Loss of CC3/TIP30 allows tumor cells to cope with low glucose. Cell Cycle 10: 376, 2011.

16. Nakahara J, Kanekura K, Nawa M, Aiso S and Suzuki N: Abnormal expression of TIP30 and arrested nucleocytoplasmic transport within oligodendrocyte precursor cells in multiple sclerosis. J Clin Invest 119: 169-181, 2009.

17. Herbst RS and Shin DM: Monoclonal antibodies to target epidermal growth factor receptor-positive tumors: A new paradigm for cancer therapy. Cancer 94: 1593-1611, 2002.

18. Humphrey PA, Wong AJ, Vogelstein B, Friedman HS, Werner MH, Bigner DD and Bigner SH: Amplification and expression of the epidermal growth factor receptor gene in human glioma xenografts. Cancer Res 48: 2231-2238, 1988.

19. Zhang C, Li A, Zhang X and Xiao H: A novel TIP30 protein complex regulates EGF receptor signaling and endocytic degradation. J Biol Chem 286: 9373-9381, 2011.

20. Louis DN, Ohgaki H, Wiestler OD, Cavenee WK, Burger PC, Jouvet A, Scheithauer BW, Kleihues P: The 2007 WHO classification of tumours of the central nervous system. Acta Neuropathol 114: 97-109, 2007.

21. Shi M, Yan SG, Xie ST and Wang HN: Tip30-induced apoptosis requires translocation of Bax and involves mitochondrial release of cytochrome $c$ and Smac/DIABLO in hepatocellular carcinoma cells. Biochim Biophys Acta 1783: 263-274, 2008.

22. Zhao J, Lu B, Xu H, Tong X, Wu G, Zhang X, Liang A, Cong W, Dai J, Wang H, et al: Thirty-kilodalton Tat-interacting protein suppresses tumor metastasis by inhibition of osteopontin transcription in human hepatocellular carcinoma. Hepatology 48: 265-275, 2008.

23. Jiang C, Pecha J, Hoshino I, Ankrapp D and Xiao H: TIP30 mutant derived from hepatocellular carcinoma specimens promotes growth of HepG2 cells through up-regulation of N-cadherin. Cancer Res 67: 3574-3582, 2007.

24. Halatsch ME, Schmidt U, Behnke-Mursch J, Unterberg A and Wirtz CR: Epidermal growth factor receptor inhibition for the treatment of glioblastoma multiforme and other malignant brain tumours. Cancer Treat Rev 32: 74-89, 2006.

25. Zhang C, Mori M, Gao S, Li A, Hoshino I, Aupperlee MD, Haslam SZ and Xiao H: Tip30 deletion in MMTV-Neu mice leads to enhanced EGFR signaling and development of estrogen receptor-positive and progesterone receptor-negative mammary tumors. Cancer Res 70: 10224-10233, 2010.

26. Zhang C, Li A, Gao S, Zhang X and Xiao H: The TIP30 protein complex, arachidonic acid and coenzyme A are required for vesicle membrane fusion. PLoS One 6: e21233, 2011. 